\title{
Synchronization on Finite Precision Devices
}

\author{
Liu Lingfeng ${ }^{*}, 1$ and Miao Suoxia ${ }^{2}$ \\ ${ }^{1}$ School of Software, Nanchang University, Nanchang, Jiangxi, 330031, P.R. China \\ ${ }^{2}$ School of Science, Nanchang Institute of Technology, Nanchang, Jiangxi, 330099, P.R. China
}

\begin{abstract}
In the practical applications, most of the chaotic systems are on the finite precision devices (such as computer), and their trajectories turn to be periodic which do not meet their evolution functions. The synchronization of digital chaotic systems has been studied in this paper. Both theoretical analysis and numerical experiments show that the digital systems can achieve synchronization. The relation between the roundoff of computer and synchronization has also been discussed in this paper.
\end{abstract}

Keywords: Chaos, digital systems, finite precision, synchronization.

\section{INTRODUCTION}

Chaotic synchronization was first proposed by Pecora and Carroll in 1990 [1], they use the conditional Lyapunov exponent of the response system to judge synchronization. After this, studies show that it is only a necessary condition of synchronization. Although all the conditional Lyapunov exponents of response system are smaller than 0 , the systems may not achieve synchronization. In 1992, He and Vaidya proposed the judgment of synchronization by the state error of driven and response systems [2]. The systems can achieve synchronization if and only if the state error converges to 0 . Till now, it is still the most widely used judgment of synchronization [3-7].

Shortly after chaotic synchronization was proposed, it led to extensive study and application in secure communication. In recent years, a growing number of cryptosystems based on continuous systems utilize the idea of synchronization of chaos $[8,9]$. However, recent studies show that the performance of these systems is very poor and insecure [10, 11]. The insecurity results mainly from the insensitivity of synchronization to system parameters [12]. Recently, discrete chaotic communication systems have been given much more attention [13-15]. However, all the discrete chaotic systems are on the finite precision devices (such as computer). Trying to generate a chaotic signal on a finite precision device leads to dynamical degradation of chaotic properties [16]. The chaotic trajectories turn to be periodic in a discrete phase space. In other words, there is no chaos under finite precision [17].

Among all the studies of chaos, including chaotic synchronization, the results are all based on ideal conditions. The problem of finite precision has always been ignored in theoretical studies. Motivated by this, in this paper, we study on the synchronization of chaotic systems which are on the computers. We call them digital chaotic systems in this paper. Denote $\delta$ be the rounoff of computer, two cases have been considered: 1) The two digital systems are on the computers with the same $\delta$. 2) The two digital systems are on the computers with different $\delta$. Both theoretical analysis and experiments show that the digital chaotic systems can achieve synchronization. Furthermore, we also discuss the relation between synchronization and the roundoff of computer.

The paper is organized as follows. In Section 2, we prepare some preliminaries. The synchronization of digital chaotic systems with the same roundoff is studied in Section 3. The synchronization of digital chaotic systems with different roundoff is studied in Section 4. Finally, we close our paper with conclusions in Section 5.

\section{PRELIMINARIES}

Consider the following driven and response systems

$x_{k+1}=F\left(x_{k}, p\right)$

$y_{k+1}=G\left(y_{k}, q\right)+C\left(x_{k}, y_{k}\right), \quad k=0,1, \ldots, N-1$

Here $x_{k}$ and $p$ are state variable and parameter of the chaotic system $F$ respectively. $y_{k}$ and $q$ are state variable and parameter of the chaotic system $G$ respectively. $C\left(x_{k}, y_{k}\right)$ denotes control function for state variable. The choice of control function is not unique [18]. The synchronization of these two systems is defined as:

Definition 1. Let $e_{k}=y_{k}-x_{k}$ be the error system of the driven system (1) and the response system (2), then system (1) and (2) are said to be synchronized if

$\lim _{k \rightarrow \infty}\left\|e_{k}\right\|=0$

here $\|\cdot\|$ denotes the Euclidean norm. 
Definition 1 is the most widely used judgment of synchronization. The error $e_{k}$ is continuous and converge to zero with time $k$. Once the systems are $F$ and $G$ are implemented on a finite precision device (such as computer), the phase space of $x_{k}$ and $y_{k}$ become discrete, and the phase space of error $e_{k}$ becomes discrete. Therefore, it is not suitable to judge the synchronization of digital systems by (3).

Consider the following two digtial systems

$$
\begin{aligned}
& x_{k+1}=\left\{F\left(x_{k}, p\right)\right\} \\
& y_{k+1}=\left\{G\left(y_{k}, q\right)+C\left(x_{k}, y_{k}\right)\right\}, k=0,1, \ldots, N-1
\end{aligned}
$$

Here $\{\cdot\}$ denotes the finite precision represented. $x_{k}$ and $p$ are state variable and parameter of the digital system $F$ respectively. $y_{k}$ and $q$ are state variable and parameter of the digital system $G$ respectively. $C\left(x_{k}, y_{k}\right)$ denotes control function for state variable. The synchronization of these two digital systems is defined as:

Definition 2. Let $e_{k}=y_{k}-x_{k}$ be the error system of the driven system (4) and the response system (5), then system (4) and (5) are said to be synchronized if there exist a natural number $K$, for every $k \geq K$, we have $\left\|e_{k}\right\| \leq \varepsilon$. $\varepsilon$ is the error which can be tolerated in practical applications.

The digital systems can achieve synchronization in practical if they satisfy definition 2 . In this paper, we only consider the synchronization of the same system and parameter, that is $F=G$, and $p=q$.

\section{SYNCHRONIZATION OF DIGITAL SYSTEMS WITH THE SAME ROUNDOFF}

In this Section, we will study on the synchronization of digital chaotic systems with the same $\delta$. Theorem 1 shows that the digital systems can achieve synchronization in practical with a suitable control function $C\left(x_{k}, y_{k}\right)$.

Theorem 1. Consider the following two digital chaotic systems

$x_{k+1}=\left\{F\left(x_{k}, p\right)\right\}$

$y_{k+1}=\left\{F\left(y_{k}, p\right)+C\left(x_{k}, y_{k}\right)\right\}, k=0,1, \ldots, N-1$

Here, $x_{k}$ and $y_{k}$ are the state variables, $p$ is the parameter of system and $C\left(x_{k}, y_{k}\right)=\left(k_{1} \mathbf{I}-\left(\left(f\left(y_{k}, p\right)-f\left(x_{k}, p\right)\right)\left(y_{k}-x_{k}\right)^{-}\right.\right.$ $\left.{ }^{1}\right) \cdot\left(x_{k}-y_{k}\right)$ is the control function, $k_{1}$ is the control coefficient, and $I$ is the unit matrix. The systems (6) and (7) can achieve synchronization in practical if $\left|k_{1}\right|<1-\delta / \varepsilon$.

Proof: Subtract (6) from (7), we have

$$
\begin{aligned}
e_{k+1}= & y_{k+1}-x_{k+1} \\
= & \left\{F\left(y_{k}, p\right)+C\left(x_{k}, y_{k}\right)\right\}-\left\{F\left(x_{k}, p\right)\right\} \\
= & F\left(y_{k}, p\right)+\delta_{k, y}+C\left(x_{k}, y_{k}\right)-F\left(x_{k}, p\right)-\delta_{k, x} \\
= & F\left(y_{k}, p\right)-F\left(x_{k}, p\right)+\delta_{k, y}-\delta_{k, x} \\
& +\left(k_{1} \mathrm{I}-\left(\left(F\left(y_{k}, p\right)-F\left(x_{k}, p\right)\right)\left(y_{k}-x_{k}\right)^{-1}\right) \cdot\left(x_{k}-y_{k}\right)\right. \\
= & k_{1}\left(y_{k}-x_{k}\right)+\delta_{k, y}-\delta_{k, x}
\end{aligned}
$$

Here $\delta_{k, x}$ and $\delta_{k, y}$ are the truncation errors, and we have $\max \left\{\delta_{k, y}, \delta_{k, x}\right\}<\delta$. Then

$$
\left\|e_{k+1}\right\|<\left|k_{1}\right| \cdot\left\|e_{k}\right\|+\delta
$$

If $\left\|e_{k}\right\|>\varepsilon$ (which means before achieved synchronization), we have

$$
\left|e_{k+1}\right||<| k_{1} \mid \cdot\left\|e_{k}\right\|+\delta \cdot\left\|e_{k}\right\| / \varepsilon<\left(\left|k_{1}\right|+\delta / \varepsilon\right) \cdot\left\|e_{k}\right\|
$$

Let $A=\left|k_{1}\right|+\delta / \varepsilon$, we have $|A|<1$. Here, we can see that the error $\left\|e_{k}\right\|$ decreases with the growth of $k$. Thus, there must exist a critical value $K$, satisfy

$\left\|e_{K}-1\right\|>\varepsilon$

$\left\|e_{K}\right\|<\varepsilon$

Next, we will proof that if $\left\|y_{K}-x_{K}\right\|<\varepsilon$, then we have $\|$ $y_{K+1}-x_{K+1} \|<\varepsilon$, which means that systems (6) and (7) can achieve synchronization in practical.

$$
\begin{gathered}
\left\|e_{K+1}\right\|<\left|k_{1}\right| \cdot\left\|e_{K}\right\|+\delta<\left|k_{1}\right| \varepsilon+\delta \\
=\left(\left|k_{1}\right|+\delta / \varepsilon\right) \varepsilon<\varepsilon
\end{gathered}
$$

Thus, concluding our proof.

Remark 1. Due to the inequality $\left|k_{1}\right|<1-\delta / \varepsilon$, we have that if the tolerated error $\varepsilon$ is larger than the roundoff $\delta$, the digital systems (6) and (7) can achieve synchronization by choosing a suitable control coefficient $k_{1}$.

Next, we do some numerical experiments. Consider the following digital Logistic maps

$$
\begin{aligned}
& x_{k+1}=\left\{r x_{k}\left(1-x_{k}\right)\right\} \\
& y_{k+1}=\left\{r y_{k}\left(1-y_{k}\right)+C\left(x_{k}, y_{k}\right)\right\}
\end{aligned}
$$

The control function $C\left(x_{k}, y_{k}\right)=\left(r-r x_{k}-r y_{k}-k_{1}\right)\left(x_{k}-y_{k}\right)$.

The roundoff of the computer is $2^{-16}$. Let $r=4, k_{1}=0.2$, $x_{0}=0.3541, y_{0}=0.6325, \varepsilon=2^{-15}$, the experiment results are shown in Fig. (1). The experiments by employing different parameters and initial values indicate that this scheme is efficient and robust.

Fig. (2) shows the relation between the roundoff and the critical value $K . K$ denotes the step when the systems firstly achieve synchronization. Here $\varepsilon=2 \delta$. Fig. (2) shows that the larger the roundoff is, the slower the systems achieve synchronization. The relation is approximately linear.

\section{SYNCHRONIZATION OF DIGITAL SYSTEMS WITH DIFFERENT ROUNDOFF}

In this Section, we will study on the synchronization of digital chaotic systems with different $\delta$.

Consider the following two digital Logistic systems

$$
\begin{aligned}
& x_{k+1}=\left\{F\left(x_{k}, p\right)\right\}_{1} \\
& y_{k+1}=\left\{F\left(y_{k}, p\right)+C\left(x_{k}, y_{k}\right)\right\}_{2}, k=0,1, \ldots, N-1
\end{aligned}
$$




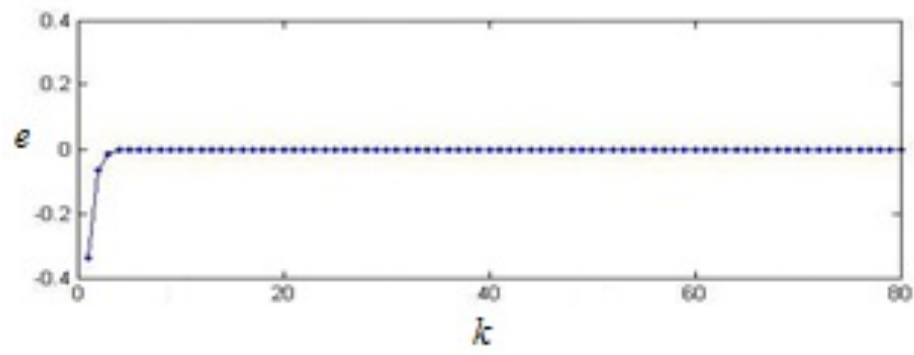

Fig. (1). Synchronization of digital Logistic map with the same roundoff

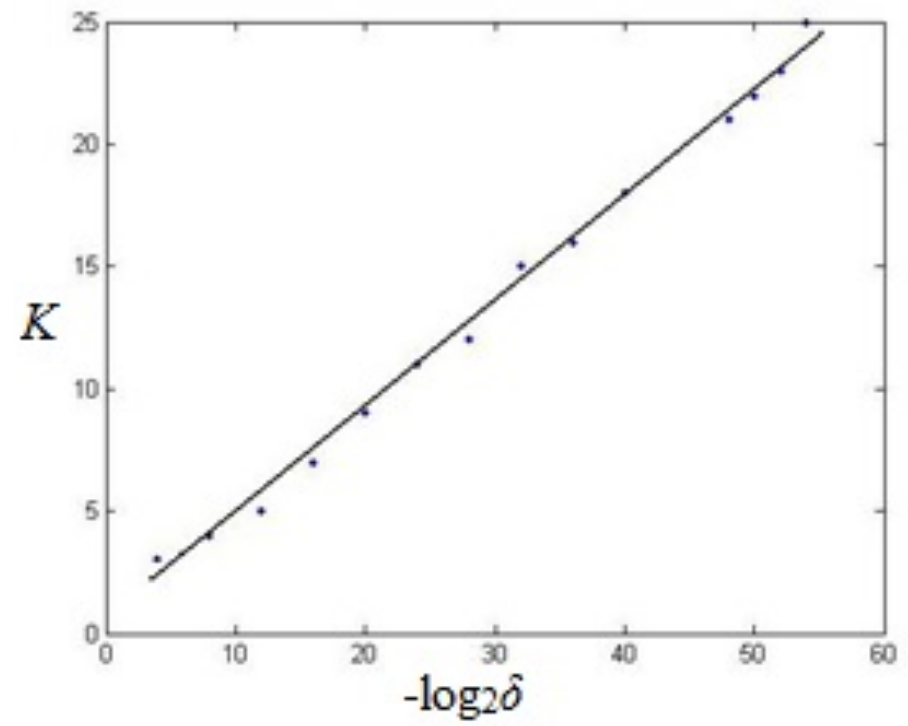

Fig. (2). The relation between the roundoff and the critical value $K$. Dots are data from the numerical experiments.

Here, $x_{k}$ and $y_{k}$ are the state variables, $p$ is the parameter of system and $C\left(x_{k}, y_{k}\right)$ is the control function. $\{\cdot\}_{1}$ and $\{\cdot\}_{2}$ denote the finite precision represented of these two systems, the roundoff are $\delta_{1}$ and $\delta_{2}$ respectively.

Eq. (10) is the driven system and Eq. (11) is the response system. The synchronization is implemented on the second computer with roundoff $\delta_{2}$. If $\delta_{1}<\delta_{2}$, the driven signal will be truncated. The driven signal will be represented with roundoff $\delta_{2}$. These two digital systems are with the same roundoff $\delta_{2}$, which has already been studied in Section 3 . Next, we consider the case of $\delta_{1}>\delta_{2}$.

Theorem 2. Consider the digital chaotic systems (10) and (11), $C\left(x_{k}, y_{k}\right)=\left(k_{1} \mathbf{I}-\left(\left(F\left(y_{k}, p\right)-F\left(x_{k}, p\right)\right)\left(y_{k}-x_{k}\right)^{-1}\right) \cdot\left(x_{k}-y_{k}\right)\right.$ is the control function, $k_{1}$ is the control coefficient, and $\mathbf{I}$ is the unit matrix, $\delta_{1}>\delta_{2}$. The systems (10) and (11) can achieve synchronization in practical if $\left|k_{1}\right|<1-\delta_{1} / \varepsilon$.

Proof: Subtract (10) from (11), we have

$$
\begin{aligned}
& e_{k+1}=y_{k+1}-x_{k+1} \\
& =\left\{F\left(y_{k}, p\right)+C\left(x_{k}, y_{k}\right)\right\}_{2}-\left\{F\left(x_{k}, p\right)\right\}_{1} \\
& =F\left(y_{k}, p\right)+\delta_{2, k, y}+C\left(x_{k}, y_{k}\right)-F\left(x_{k}, p\right)-\delta_{1, k, \mathrm{x}} \\
& =F\left(y_{k}, p\right)-F\left(x_{k}, p\right)+\delta_{2, k, y}-\delta_{1, k, \mathrm{x}}
\end{aligned}
$$

$$
\begin{aligned}
& +\left(k_{1} \mathrm{I}-\left(\left(F\left(y_{k}, p\right)-F\left(x_{k}, p\right)\right)\left(y_{k}-x_{k}\right)^{-1}\right) \cdot\left(x_{k}-y_{k}\right)\right. \\
& =k_{1}\left(y_{k}-x_{k}\right)+\delta_{2, k, y}-\delta_{1, k, \mathrm{x}}
\end{aligned}
$$

Here, $\delta_{1, k, x}$ and $\delta_{2, k, y}$ are the truncation errors, then we have $\max \left\{\delta_{1, k, x}\right\}<\delta_{1}$, and $\max \left\{\delta_{2, k, y}\right\}<\delta_{2}$, thus

$\left\|e_{k+1}\right\|<\left|k_{1}\right| \cdot\left\|e_{k}\right\|+\delta_{1}$

If $\left\|e_{k}\right\|>\varepsilon$ (which means before achieved synchronization), we have

$$
\left\|e_{k+1}\right\|<\left|k_{1}\right| \cdot\left\|e_{k}\right\|+\delta_{1} \cdot\left\|e_{k}\right\| / \varepsilon<\left(\left|k_{1}\right|+\delta_{1} / \varepsilon\right) \cdot\left\|e_{k}\right\|
$$

Let $A=\left|k_{1}\right|+\delta_{1} / \varepsilon$, we have $|A|<1$. Here, we can see that the error $\left\|e_{k}\right\|$ decreases with the growth of $k$. Thus, there must exist a critical value $K$, satisfy

$$
\begin{gathered}
\left\|e_{K}-1\right\|>\varepsilon \\
\left\|e_{K}\right\|<\varepsilon
\end{gathered}
$$

Next, we will proof that if $\left\|y_{K}-x_{K}\right\|<\varepsilon$, then we have $\|$ $y_{K+1}-x_{K+1} \|<\varepsilon$, which means that systems (10) and (11) can achieve synchronization in practical.

$$
\left\|e_{K+1}\right\|<\left|k_{1}\right| \cdot\left\|e_{K}\right\|+\delta_{1}<\left|k_{1}\right| \varepsilon+\delta_{1}
$$




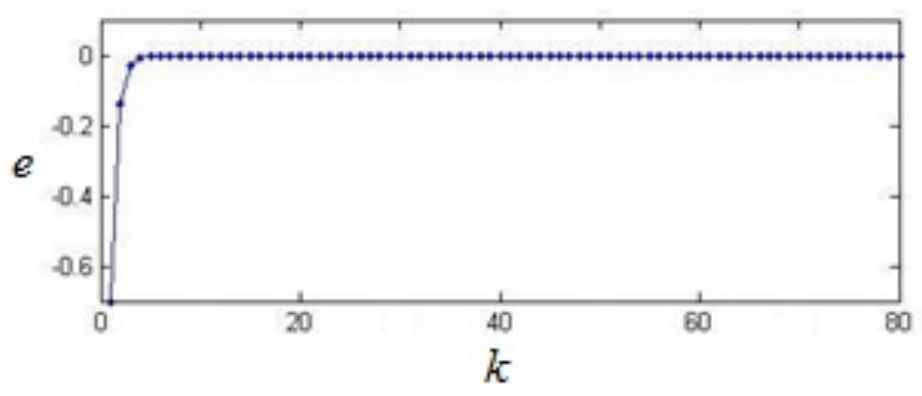

Fig. (3). Synchronization of digital Logistic map with different roundoff.

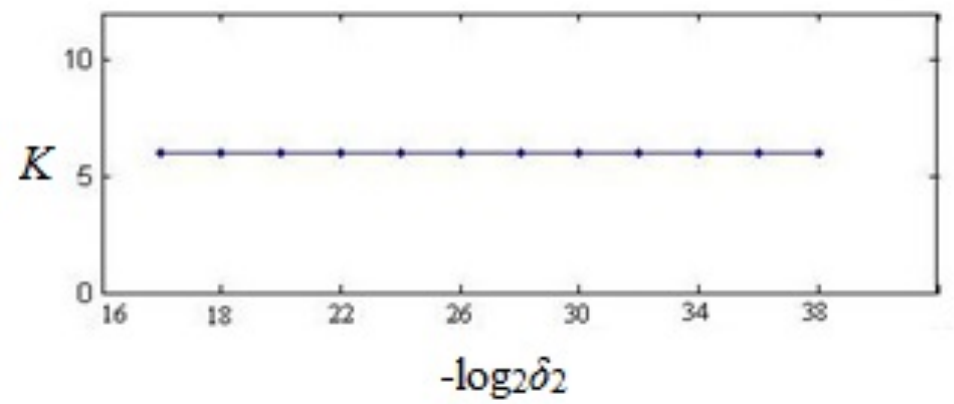

Fig. (4). The curve of $K$ when $\delta_{2}$ changes. Dots aredata from the numerical experiments.

$=\left(\left|k_{1}\right|+\delta_{1} / \varepsilon\right) \varepsilon<\varepsilon$

Thus, concluding our proof.

Remark 2: Due to the inequality $\left|k_{1}\right|<1-\delta_{1} / \varepsilon$, we see that the synchronization is only influenced by $\delta_{1}$. For any $\varepsilon>$ $\delta_{1}$, the digital systems (10) and (11) can achieve synchronization by choosing a suitable control coefficient $k_{1}$.

Also, we do some numerical experiments. Consider the following two digital Logistic maps

$x_{k+1}=\left\{r x_{k}\left(1-x_{k}\right)\right\}_{1}$

$y_{k+1}=\left\{r y_{k}\left(1-y_{k}\right)+C\left(x_{k}, y_{k}\right)\right\}_{2}$

The control function $C\left(x_{k}, y_{k}\right)=\left(r-r x_{k}-r y_{k}-k_{1}\right)\left(x_{k}-y_{k}\right)$.

The roundoff of the computer are $2^{-16}$ and $2^{-24}$ respectively. Let $r=4, k_{1}=0.2, x_{0}=0.1, y_{0}=0.8, \varepsilon=2^{-15}$, the experiment results are shown in Fig. (3). The experiments by employing different parameters and initial values indicate that this scheme is efficient and robust.

From theorem 2 and the analysis above we know, the synchronization of digital systems mainly depends on the computer which has a larger roundoff. Let $\delta_{1}=2^{-16}$ and $\varepsilon=2^{15}$, Fig. (4) shows that the critical value $K$ remains the same when $\delta_{2}$ changes.

\section{CONCLUSION}

The digital chaotic systems on the finite precision devices is not chaotic any more, their trajectories turn to be periodic. In this paper, we focus on the digital systems, discuss the synchronization of them. The theorems and experiments in this paper show that the digital systems can achieve synchronization with both the same and different roundoff. The relation between the roundoff and the synchronization speed has also been studied. Besides synchronization, other studies on digital chaotic system are also of great interest, such as digital (discrete) chaos theory. In our future work, we will study on this issue.

\section{CONFLICT OF INTEREST}

The authors confirm that this article content has no conflict of interest.

\section{ACKNOWLEDGEMENTS}

Declared none.

\section{REFERENCES}

[1] L. M. Pecora and T. L. Carroll, "Synchronization in chaotic systems," Physical Review Letters, vol. 64, pp. 821-824, Feb 1990.

[2] R. He and P. G. Vaidya, "Analysis and synthesis of synchronous periodic and chaotic systems," Physical Review A, vol. 46, pp. 7387-7392, Dec, 1992.

[3] R. Z. Luo and Z. M. Wei, "Adaptive function projective synchronization of unified chaotic systems with uncertain parameters," Chaos, Solitons and Fractals, vol. 42, pp. 1266-1272, Oct, 2009.

[4] D. Li, Z. D. Wang, J. Zhou, J. A. Fang and J. J. Ni, "A note on chaotic synchronization of time-delay secure communication systems, Chaos, Solitons and Fractals, vol. 38, pp. 1217-1224, Nov, 2008 .

[5] C. K. Zhang, Y. He and M. Wu, "Improved global asymptotical synchronization of chaotic Lur'e systems with sampled-data control," IEEE Transactions on Circuits and Systems II: Express Briefs, vol. 56, pp. 320-324, Apr, 2009.

[6] J. H. Jiang, Y. Xin, T. S. Lande and Y. Lian, "An efficient synchronization scheme for digital UWB communication systems for biomedical applications," Biomedical Circuits and Systems Conference, BioCAS 2008, IEEE, pp. 13-16, 2008 
[7] Z. Q. Zhu and H .P .Hu, "Robust synchronization by time-varying impulsive control," IEEE Transactions on Circuits and Systems II: Express Briefs, vol. 57, pp. 735-739, Sep, 2010.

[8] L. Kocarev and U. Parlitz, "General approach for chaotic synchronization with applications to communication," Physical Review Letters, vol. 74, pp. 5028-5031, Jun, 1995.

[9] S. Boccaletti, J. Kurths, G. Osipov, D. L. Valladares and C. S. Zhou, "The synchronization of chaotic systems." Physics Reports, vol. 366, pp. 1-101, Aug, 2002.

[10] K. M. Short and A. T. Parker, "Unmasking a hyperchaotic communication scheme," Physical Review E, vol. 58, pp. 1159-1162, Jul, 1998.

[11] C. Zhou and C. H. Lai, "Extracting messages masked by chaotic signals of time-delay systems," Physical Review E, vol. 60, pp. 320-323, Jul, 1990.

[12] S. Wang, J. Kuang, J. Li, Y. Luo, H. Lu and G. Hu, "Chaos-based secure communications in a large community," Physical Review E, vol. 66, p. 065202, Dec, 2002.
[13] F. Huang and Z. H. Guan, "Cryptosystem using chaotic keys," Chaos, Solitons and Fractals, vol. 23, pp. 851-855, Feb, 2005.

[14] K. W. Wong, S. W. Ho and C. K. Yung, "A chaotic cryptography scheme for generating short ciphertext," Physics Letters A, vol. 310, pp. 67-73, Apr, 2003.

[15] M. S. Baptista, "Cryptography with chaos," Physics Letters A, vol. 240, pp. 50-54, Mar, 1998

[16] S. Li, G. Chen and X. Mou, "On the dynamical degradation of digital piecewise linear chaotic maps," International Journal of Bifurcation and Chaos, vol. 15, pp. 3119-3151, Oct, 2004.

[17] L. Kocarev, J. Szczepanski, J. M. Amigo and I. Tomovski, "Discrete chaos-I: Theory," IEEE Transactions on Circuit and Systems, vol. 53, pp. 1300-1309, Jun, 2006.

[18] M. K. Ali, "Synchronization of a chaotic map in the presence of common noise," Physical Review E, vol. 55, pp. 4804-4805, Apr, 1997.

(C) Lingfeng and Suoxia; Licensee Bentham Open.

This is an open access article licensed under the terms of the Creative Commons Attribution Non-Commercial License (http://creativecommons.org/licenses/by-nc/3.0/) which permits unrestricted, non-commercial use, distribution and reproduction in any medium, provided the work is properly cited. 